\title{
XXVIII. On the contraction which takes place in mercury at low temperatures by abstraction of heat;-and on the ratio of contraction between mercury, alcohol, water, and silver
}

\author{
John Biddle Esq.
}

To cite this article: John Biddle Esq. (1808) XXVIII. On the contraction which takes place in mercury at low temperatures by abstraction of heat;-and on the ratio of contraction between mercury, alcohol, water, and silver , Philosophical Magazine Series 1, 30:118, 134-153, DOI: 10.1080/14786440808563787

To link to this article: http://dx.doi.org/10.1080/14786440808563787

$$
\text { 曲 Published online: } 18 \text { May } 2009 .
$$

Submit your article to this journal ए

\section{山 Article views: 2}

Q View related articles ¿ 


\section{On the Contraction which takes place in Mercury}

while cold, or assisted by the steam when it becomes hot, it is instantly condensed into an impalpable powder, possessing all the qualities of calomel in its most perfect state. The calomel; when thus prepared, is purer, whiter; and more attenuated, than that obtained by grinding. It is proper to wash the produot over with water, before it is dried, to rid it of any coarser particles which may form about the mouth of the crucible.

XXVIII. On the Contraction which takes place in Mercury at low Temperalures ly Alstraction of Heat; -and on the Ratio of Contraction between Mercury, Alcohal, Water, and Silver. By JoHn Brddue, Esq. of Birmingham.

Birningham, February, 1808.

\section{To Mr. Tilloch.}

A

SIR,

A Letren from M. Tardy de la Brossy, dated Joyeuse, (Ardeche) October 13th, 1805, addressed to Professor Pictet, of Geneva, has appeared in your Mayazine [vol. xxiv. p. 322]. It contains observations on some experiments which I had the pleasure of showing to the Philosophical Society here on the specific gravity of mercury in its frozen state, which experiments were cotnmunicated to the public through the medium of Mr, Nicholson's Journal for April 1805. The observations of M. Tardy de la Brossy bave induced me to look over the original, papers, containing the results of those experiments, with some attention; and, with deference to the opinions of that gentlcman (though I fear some inaccuracy, exists), 1 must, in defence of my. experiments generally, and the deductions made from them, .request you to. communicate a fow experiments and observations through the chaninel of your Magazine.

M. Tandy de la Brossy, after avowing the object of his communication to be "the extension of truth, and the removal of error," proceeds to describe the result of some of my formei experiments, and to make his observations on my calculations from them, to which I would refer; but if 
he had added experiment to his calculations, he would have been convinced that the principles on which I proceeded are just, and that one source of difference in our opinions arises from the partial application of the mode of reasoning which he uses. For, adnitting with him the specific gravity of the alcohol employed to be 810 nearly, or $\cdot 8141$ where water is $1 \cdot 000$, and that 1000 grains of mercury would exhibit a loss of weight in alcohoi of the temperature stated, of $59 \cdot 8$, when weighed by the hydrostatic balance, yet it does not appear to me necessary to suppose, a priori, so long as each of these substances remains in a fluid state, that the ratio of their densities should differ when uniformly subjected to the lower degrees of heat. As, however, my former experiments were not made with a view to discover the contraction of the volume of the alcohol, I made, no observation relating to it, and now think it right to investigate the subject by experiment.

A. Ifirst distilled mercury as before, with great care, using only that 30 per cent. of the whole which first came over in the distillation, estecming it the most pure. I found, by the hydrostatic balance, the specific gravity of this to be 13.613 , as 1000 grains lost in distilled water 73.4 at the temperature 50 of Fahrenheit's scale.

B. I took alcohol from the same parcel which I had used in my former experiments, and filling a light glass bottle formed with a long narrow neck for the purpose, it was found to weigh 8141 , when water weighed. 1.000 at the temperature of $48^{\circ}$ nearly.

C. Having obtained a mass of very pure silver, procured from luna cornea, $1000 \mathrm{gr}$. lost in distilled water $97 \cdot 8$, the specific gravity of which was thereby found to be 10.225 ; but by hambering it into a form convenient for my purpose the specific gravity increased to 10.362 , the loss of weight being 96.5 at $50^{\circ}$ of temperature. At. the following temperatures the variations of loss, of weight are expressed in the secand column, the consequent specific gravities in the third, according to the usual mode of calculation, and in the fourth is shown the loss of weight in alcohol:-parts of a series of observations from experments, that are given more 


\section{On the Contraction which takes place in Mercury}

at large in the table containing a general comparison of the several experiments.

\begin{tabular}{|c|c|c|c|}
\hline 1 & 2 & 3 & 4 \\
\hline $\begin{array}{l}\text { Degrees of hea } \\
\text { according to } \\
\text { Fahrenheit's } \\
\text { scale. }\end{array}$ & $\begin{array}{c}\text { Loss of weight } \\
\text { of } 1000 \mathrm{gr} \text {. of } \\
\text { silverin distilled } \\
\text { water. }\end{array}$ & $\begin{array}{l}\text { Specific gravity } \\
\text { of silver when } \\
\text { water is } 1 \cdot 000 \text {. }\end{array}$ & $\begin{array}{l}\text { Loss of weight } \\
\text { of } 1000 \text { gr.of sil- } \\
\text { ver weighed in } \\
\text { alcohol. }\end{array}$ \\
\hline 200 & 94 & $10 \cdot 638$ & \\
\hline 185 & $94 \cdot 4$ & $10 \cdot 593$ & \\
\hline 175 & $94 \cdot 6$ & 10.570 & \\
\hline 158 & $95 \cdot 2$ & $10 \cdot 504$ & \\
\hline 150 & $95 \cdot 3$ & $10 \cdot 493$ & \\
\hline 130 & $95 \cdot 5$ & $10 \cdot 471$ & $75 \cdot 4$ \\
\hline 102 & $95 \cdot 9$ & $10 \cdot 428$ & $76 \cdot 2$ \\
\hline 80 & $96 \cdot 3$ & $10 \cdot 384$ & $77 \cdot 6$ \\
\hline 66 & $96 \cdot 4$ & $10 \cdot 373$ & $78 \cdot 5$ \\
\hline 55 & $96 \cdot 4$ & $10 \cdot 373$ & \\
\hline 50 & $96 \cdot 5$ & $10 \cdot 362$ & $78 \cdot 6$ \\
\hline 34 & $96 \cdot 5$ & $10 \cdot 362$ & $79 \cdot 4$ \\
\hline 21 & - & - & 80 \\
\hline 9 & - & - & $80 \cdot 5$ \\
\hline o & - & 一 & 81 \\
\hline 20 & - & - & $81 \cdot 6$ \\
\hline 32 & - & - & $82 \cdot 5$ \\
\hline 54 & - & - & 83 \\
\hline 56 & - & - & $83 \cdot 1$ \\
\hline
\end{tabular}

D. By the following experiments $I$ then proceeded to. ascertain the specific gravity of mercury and silver at various temperatures, from $100^{\circ}$ above zero to more than $40^{\circ}$ below it, with a wish to obtain a ratio of contraction between mercury and silver, and between each of these and alcohol. For this purpose two hydrostatic balances were so placed that the metals suspended from them might fall into a glass vessel containing alcohol.

I then attached 1000 grains of silver to one balance, by a wire 12 inches long, weighing only $\frac{7}{10}$ of a grain; and to the other 1000 grains of mercury, in a small glass bucket weighing 101 To grains, suspended by a wire nearly 4 inches long, weighing $\frac{2}{10}$ grain.

Then also suspending in the alcohol a good thermometer, marked 
marked for low temperatures, and having provided a quantity of muriate of lime and snow, I reduced the temperature of the alcohol and metals, as in my former experiments the subject of M. Tardy de la Brossy's observations, and obtained the results expressed in the following table.

\begin{tabular}{|c|c|c|c|c|c|c|}
\hline 1 & 2 & 3 & 4 & 5 & 6 & 7 \\
\hline 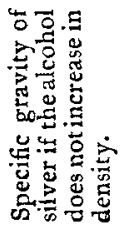 & 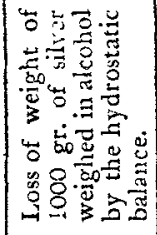 & 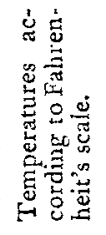 & 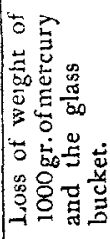 & 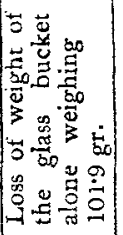 & 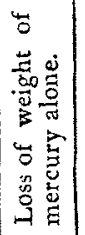 & 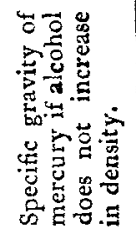 \\
\hline $10 \cdot 680$ & $76 \cdot 2$ & 100 & $82 \cdot 6$ & $24 \cdot 3$ & $58 \cdot 3$ & 13.963 \\
\hline 10.518 & $77 \cdot 4$ & 60 & $85 \cdot 0$ & $85 \cdot 3$ & $59 \cdot 7$ & $13 \cdot 63 t i$ \\
\hline $10 \cdot 362$ & 78.56 & 50 & $85 \cdot 3$ & $25 \cdot 5$ & $59 \cdot 8$ & 13.613 \\
\hline $10 \cdot 359$ & $78 \cdot 3$ & 44 & $85 \cdot 5$ & $25 \cdot 6$ & $59 \cdot 9$ & $13 \cdot 589$ \\
\hline $10 \cdot 208$ & $79 \cdot 7$ & 20 & $86 \cdot 6$ & 26 & $60 \cdot 6$ & 13.433 \\
\hline $10 \cdot 290$ & $79 \cdot 9$ & 6 or 7 & $86 \cdot 7$ & $26 \cdot 1$ & $60 \cdot 6$ & I $3 \cdot 433$ \\
\hline 10.037 & $\cdot 1$ & 0 & $86 \cdot 9$ & $26 \cdot 2$ & $60 \cdot 7$ & 13.411 \\
\hline
\end{tabular}

In an examination of the 1 st and $2 \mathrm{~d}$ columns of this table relating to silver, it being previously known that 10:362 is the specific gravity of silver, and that .8141 is the specific gravity of alcohol at $50^{\circ}$ of Fahrenheit, where water is 1.000 , it appears, following the general mode of calculation in which the loss of weight is made the divisor of the quantity weighed, and the quotient expresses the specific gravity, that as at 100 degrees of heat the divisor for the quantity of metal is less, and at zero greater, that at zero the specific gravity is less, and at 100 degrees above it the specific gravity is greater:-A conclusion opposing a general law, which therefore we cannot admit.

To account for this apparent inaccuracy of result, we must direct our attention to the alcohol employed, and see if, in the comparison of the fluid alcohol and the solid silver, this appearance of contradiction to an established law does not arise from the contraction of the fluid. Of this it will be difficult to bring visible proof to the extent required; for if I put into a glass tube alcohol, of which the weight is 


\section{On the Contraction which takes place in Mercury}

known, and expose it to difierent temperatures from 100 to 50 degrees, it will eontract; but whilst the liuid contracts, the glass which contains it contracts also, and shows only the difference of the contraction between the fluid, and glass :-however, as we know that silver by. heating cannot become specifically heavier, or by cooling specifically lighter, in these experiments which are evidenuly a comparison of densities, the alcohol must become more dense by deprivation of beat.

The third column of this table contains the temperatures.

In the 4th column the $1000 \mathrm{gr}$. of mercury and the glass bucket containing it appear together to have varied at the two extremës in loss of weight $4 \cdot 3$.

By the 5th column it is shown that the glass bucket alone varied at the two extrethes in loss of weight 1:9.

By the 6th column it appears that $2 \cdot 4$ only can be stated to belong indispensably to a variation of the respective densities of the mercury and alcohol in the clianges of $\mathrm{km}$ perature from $100^{\circ}$ to zero, The other variations, I presume, arise from some inaccuracy, such as making the observitions when the fluids were at different temperatures.

By the 7 th column are shown the difierent sperific gravities of the mereury, by calculation from the loss of weight, supposing the density of the alcohol the same throughout : from this it appears that the density of mercury is greatest at the highest temperature, and least at the lowest; but as this cannot be actually the case, it is obvious that the alcobol increases in density; and this appears also from the results of the ed column relating to silver, at $C$.

For the specific gravities of alcohol at various temperatures, I must refer to the calculations at $I_{\text {, }}$. matle from the following experiments, and which are containcd also in a general table of results.

E. I procured the bladder of a rabbit, and washed it well with alcohol. It weighed 7 grains in air; and having annealed a wire about 9 inches long, the weight of which was $i^{6}$ ths of a grain in air, I found that the bladder and wire, when sunk in alcobol to a certain mark on the wire, weighed Q.5 grains only, at the temperature $45^{\circ}$. 
This bladder was filled with mercury, not quite pure, then suspended by the wire; and when one end of the wire was attached to the balance, it was found to contain $\mathbf{5 2 7 6}$ grains; weighed in alcohol, it lost 321.5 , from which deducting the weight of the bladder and wire in alcohol, 2.5 , it appears that the loss of the 5276 grains of mercury, when the thermometer is suspended in the alcohol

at $45^{\circ}$ of temperature, is 319

65 the mercury loses 315 less by 4

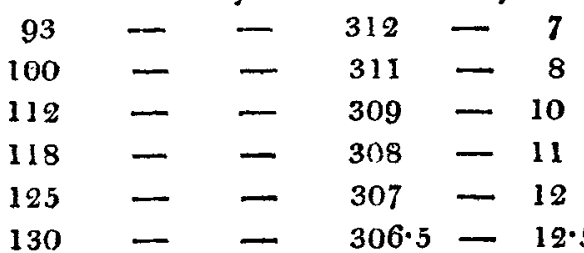

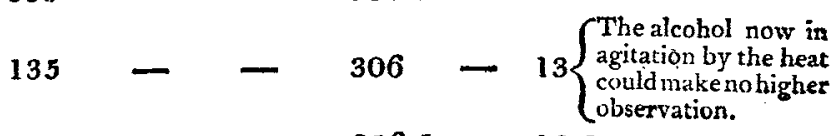

$130-\quad-306.5-12.5$

$125-307-12$

$123-307.5-11.5$

$120-308-11$

$118-308.5-10.5$

$117-309-10$

$113-309.5-9.5$

$111-310-9$

$108-310.5-8.5$

$104-314-8$

$100-311.5-7.5$

$97-312-7$

$93-\quad-312.5-6.5$

$89-313-6$

$85-313.5-5.5$

$83-514-5$

$80-315-4$

$78-316-3$

$76-317-2$

$70-317.5-1.5$

$68-318-1$ 
140 On the Contraction which takes place in Mercury

\begin{tabular}{|c|c|c|c|c|c|}
\hline 62 & - & - & 319 & - & $\mathbf{0}$ \\
\hline 50 & - & - & $320 \mathrm{~m}$ & bre by & 1 \\
\hline 45 & - & - & $320 \cdot 5$ & - & 1.5 \\
\hline 40 & - & - & 321 & - & 2 \\
\hline 36 & - & - & $321 \cdot 5$ & - & $2 \cdot 5$ \\
\hline 34 & - & - & 322 & - & 3 \\
\hline 27 & - & - & 323.5 & - & $4: 5$ \\
\hline 14 & - & - & 324 & - & 5 \\
\hline 5 & - & - & $324 \cdot 5$ & - & $5 \cdot 5$ \\
\hline $\mathbf{o}$ & - & - & $325 \cdot 5$ & - & $6 \cdot 5$ \\
\hline \multicolumn{3}{|c|}{5 below zero } & $326 \cdot 5$ & - & $7 \cdot 5$ \\
\hline 18 & - & - & $327 \cdot 5$ & - & $8 \cdot 5$ \\
\hline 21 & - & - & 328 & - & \\
\hline
\end{tabular}

We may observe in this experiment that some difference is occasioned by the direction in which the heat passes, whether from without to the alcohol, and thence to the mercury, as in the first part of these observations from $45^{\circ}$ to $135^{\circ}$; or from within from the mercury to the alcohol, as from $135^{\circ}$ to zero and $21^{\circ}$ below : and it appears that the temperature was not the same in these two fluids when the observations were made; for when I weighed the same mercury with a hole in the bladder to introduce the bulb of a thermometer, that I might observe the variation of loss of weight when the alcohol and mercury were at the same temperature exactly, it showed the mean of these variations to be correct. So that in comparing 5276 parts of mercury with alcobol, there is a variation in the contraction of these two fluids expressed by 22 of loss of weight in passing through 156 degrees of temperature. In comparing 1000 parts of mercury with alcohol, it follows that there is a variation of loss of weight in 156 degrees of temperature expressed by $4 \cdot 17$, which shows $\cdot 0267$ is the loss of alcohol in each degree greater than that of mercury.

If these two fluids were to contract by deprivation of heat in the ratio of their specific gravities, it is obvious that the loss of weight shown by the hydrostatic balance would be the same through all the changes of temperature, the changes being the same in both fluids at the time of observation. 
It is therefore of material consequence to th:s inquiry to know in what degree equal bulks of mercury and alcohol increase together, without the hydrostatic balance denoting the alteration by any change of loss of weight; for this purpose we must look to $\mathrm{D}$, and forward to the experiment $\mathbf{G}$, and the calculations from them at $\mathbf{L}$ and $I$, where we shall find a rule for discovering the specific gravity of alcohol.

F. To find the proportion of contraction between mercury and water, I took 3024 grains of mercury in a bladder, as before; and weighing it in distilled water by the hydrostatic balance, the mercury and water heated to the temperature

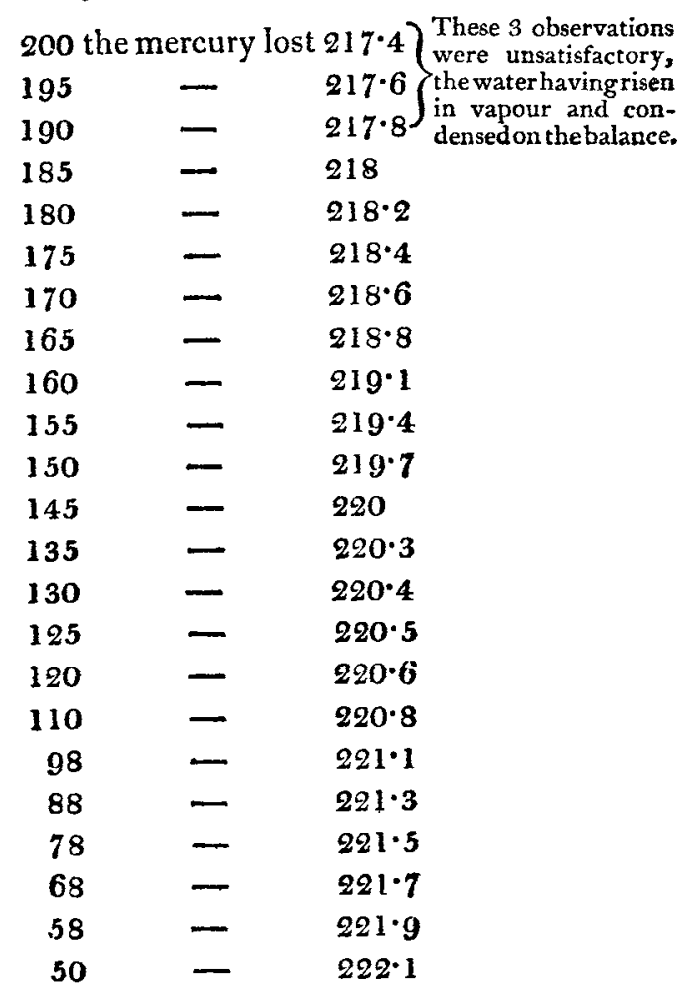

Thus, at $200^{\circ}$ of temperature mercury would appear of greater specific gravity than when at $50^{\circ}$, following the generai mode of calculation; but if we suppose the water is more 


\section{On the Contraction which takes place in Mercury}

contracted than mercury by abstraction of heaf, and attribute to that the difference of loss of weight, then, in comparing $\mathbf{3 0 2 4}$ of mercury with water, thene is a variation in the contraction of these two fluids expressed by $4 \cdot 7$ loss of weight in passing through 150 degrees of temperature. In comparing 1000 of mercury with water, a variation of loss in $150^{\circ}$ is expressed by 1.5542 .

G. The following experiment was made before many of my philosophical friends with mercury, described before, of the specific gravity of $13 \cdot 613$, suspended by a very fine wire 10 inches long, weighing only $\frac{6}{10}$ of a grain;- the barnmeter standing at $29^{\circ} 8$, the thermometer at 35 .

1000 grains of mercury with 750 grainis of alcohol, as before described, were put into a thin glass vessel, made for the purpose, ronnd at the bottom, and increasing in diameter gradually to near the top, so that the mercury might be easily suspended in the alcohol by a wire introduced into it whilst fluid. These were placed in the centre of a mixture of 4 pounds of snow and 4 pounds of muriate of lime, at 12 o'clock at noon: at 5 minutes past a thermometer placed in the frigorific mixture fell to $52^{\circ}$ below $z$ ero, then to 54 and to 60 . The mercury in the tube of the thermometer appeared frozen: it was withdrawn, and when exposed to the air a few seconds, suddenly fell to $140^{\circ}$ on the scale, in consequence of the mercury in the tube again becoming fluid, and occupying the vacuum which häd been occasioned by the contraction of the mercury in the bult after that which was in the tube had become solid : it was then immediately returned to its place in the mixture of snow and muriate of lime ; it had remained at $140^{\circ}$ for several minutes when taken out and exposeci to the air, so that the mercury still in the tube was again made fluid; it instantaneously sunk into the bulb much below $270^{\circ}$, the lowest point on the scale. During this time the mercury and alcohol, very much reduced in temperature, were removed from the above mixture, and placed in a second mixture of 3 pounds of snow and 3 pounds of muriate of lime : the whole was then placed in the first mixture; and at the moment of the crystallization of the mercury, the wire, already partly attached, was by 
raising it gently, drawn from the side to the centre of the surface of the mercury. When it was fixed and the whole was solid, it became necessary to withdraw the glass, and expose it a moment to the air of the room, until that part of the mercury attached to the glass was softened; then, by keeping one hand drawing gently at the wire, the whole of the mercury was suspended, and, with the alcohol, immediately returned to its place in the cooling mixture; the mercury was now suspended from the hydrostatic balance by the wire fixed in it, and weighed with great accuracy; - and the following observations were made from the time the second quantities of muriate of lime and snow were mixed.

In 5 minutes the mercury was crystallizing.

10

30

40

50 weighed by the hydrostatic balance with great care, lost 60.8

60 minutes the mercury and alcohol having been a little withdrawn, the mercury lost 60

85 minutes the mercury lost 59.9 it was now so nearly fuid, there was splendour on the surface.

130 minutes the mercury lost 60.8

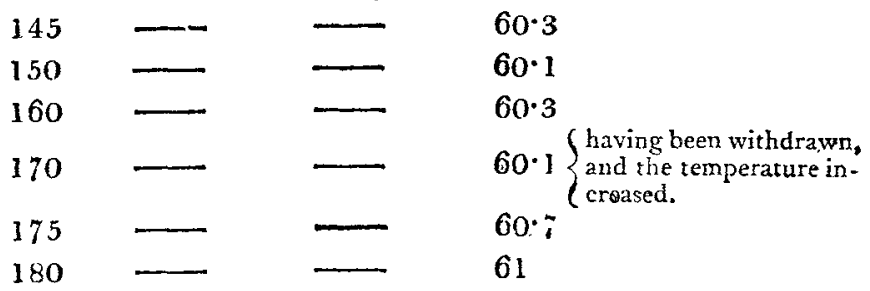

As 1 could not decrease the beat so as to indicate a greater loss than 61 , and my own body during these three hours having suffered an unusual and partial deprivation of heat, I withdrew the mercury and alcohol, not doubting, if it could have answered any purpose, by these means to have been able to have kept the mercury solid some hours longer, the-surrounding substances having lost so much heat.

We see from the observations made in this experiment, that 


\section{On the Contraction which takes place in Mercury}

59.9 is the least diminution in the weight of mercury when weighed in a solid state in alcohol, and that it is at this moment when their specific'gravilies are furthest from each other; for we may observe that at almost the next degree of temperature to that in which the mercury loses its fluidity, whilst the alcohol preserves its fluid form, the mercury appears to become of less specific gravity, as it loses 60 ; which gives, according to calculation at $L$, only 14 ; but the fact appears to me to be, that alcohol proceeds in the ratio of a fluid by decrease of temperature, and that mercury, having obtained a solid form, follows the ratio of contraction of a solid; therefore their densities approach each other. I should have ascribed this greater loss of weight to the particles of the mercury at the moment of crystallization occupying, in consequence of their new arrangement, more space than at the roment before it became solid, as with some of the metals is known to be the case, had I not carefully observed the passage of this metal in other experiments, where $I$ had an opportunity of seeing the contraction proceed very distinctly; and had not the mercury, also proceeding to still lower temperatures, lost from $60,60 \cdot 1,60 \cdot 3,60 \cdot 7,60 \cdot 8$, and 61 , long after the whole had become solid, and was suspended by the wire: thus, 61 is the greatest diminution of weight by the abstraction of heat which I could obtain, and I am of opinion that the space described by these changes of loss of weight denotes a range of many degrees of temperature: how much greater the density may then be than that stated at $\mathrm{L}$, I cannot presume to say; but as it takes, with some probability, a proportion of contraction approaching to that of silver, it certainly appears improbable that it should reach the specific gravity which my former calculation from a single observation led me to attribute to it. 1 am therefore inclined to believe there was some inaccuracy in weighing the silver by the hydrostatic balance, when by my former experiments I gave $88 \cdot 1$, the quantity of loss of $1000 \mathrm{gr}$, of silver:-from many observations in this laborious train of inquiry, it seems hardly possible that the former could have been correct ; for if the alcohol and silver continued to contract in the same proportion from $56^{\circ}$ below zero, as they do from $64^{\circ}$ above zero to that temperature, it would appear that an abstraction of heat must necessarily have 
taken place equal to $120^{\circ}$ more, or to $176^{\circ}$ below zero, which I can hardly suppose, though the mercurial thermometer used in these experiments fell lower than $270^{\circ}$ below zero. If my former statement be erroneous, it certainly is not attributable to the mode of calculation, as apprehended by M. Tardy de la Brossy. As it does not appear that he had made any experiment -on the subject, he could not have anticipated this inaccuracy in the weight of silver : and as he says that only a few grains of the increased gravity of the mercury was attributable to the alcohol, he could not be aware of the great increase of the specific gravity of alcohol shown at $\mathrm{I}$, by the same mode of calculation used in my essay, the subject of his animadversions.

It is still, however, obvious, that mercury would not be of the specific gravity which I attributed to it, unless it had followed the same rate of contraction after it became solid as it did whilst fluid : the evidence of the increased loss of weight is so much against ne, that $I$ cannot defend that experiment; and I would now be understood to carry my observations on the specific gravity of mercury, with accuracy, to the point of congrelation only; or to state that near $56^{\circ}$ below zero its specific gravity is $14 \cdot 455$, as by calculation at $L$. Then, if we attribute to mercury in a solid state nearly double the contraction of silver as at $\mathrm{H}$, or $\cdot 00100 \mathrm{in}$ each degree, as it is near to its point of fluidity, we shall arrive only at the specific gravity $14 \cdot 485$, as is shown at $\mathrm{M}$.

To ascertain whether the silver at $C$, with which I proposed to compare the mercury, had expanded or contracted by the deprivation of heat to which it had been exposed,

II-I touk an ingot of silver, 16 inches long, 2 inches wide, and half an inch thick, weighing nearly 100 ounces; and provided an instrument for the purpose of measuring accurately the contraction and expansion of the silver, by fixing in a piece of well baked wood two centre pins, exactly 15 inches from each other, one of them very fine, for the purpose of striking an arch of a circle on the surface of the silver, when the other was fixed in a pertoration made in the silver by the centre itself. Then, on the surface of the bar of silver, heated to a pale red, an arch was described with

Vol. 30. No. 118. March 1808.

$\mathrm{K}$ this 


\subsection{On the Contraction uhich takes piace in Mercupy}

this instrument; and when again reduced to the temperature of 50 degrees, another arch was drawn:-the contraction of the bar of silver between the two points appeared to equal $\frac{3}{8}$ th part of its dimensions.

The silver was then heated to $200^{\circ}$ of temperature of Fahrenheit's scale, by boiling it in water, and gradually cooling; an arch of a circle was then struck on the surface of the silver: reducing it to $150^{\circ}$ of temperature, another arch was struck with the same unvaried centre pins, which showed evident contraction in the bar of silver: again, at $100^{\circ}$ of temperature another arch was described; and $\mathbf{z}$ fourth, at $50^{2}$, showed that the silver had contracted in the deprivation of $150^{\circ}$ of temperature $\frac{1}{3}$ th part of its length.

Then, similat parallelopipedons being to each other as the cubes of their homologous sides, the increased specific gravity is shown by multiplying the specific gravity at $50^{\circ}$, namely, 10.362 , by the cube of 341 ; and dividing it by the cube of 340 , which equals $10.4537 ; \cdot 0917$, therefore, is the difference in $150^{\prime}$ of heat; or $\cdot 0006114$ is the contraction of silver in each degree; which, from the way this experiment is performed, I call its visible contraction. From it the specific gravities at the several temperatures below are calculated :-

At $200^{\circ}$ silver is of the specific gravity 10.2702

\begin{tabular}{|c|c|c|c|}
\hline 150 & & & $10 \cdot 3000$ \\
\hline 13.5 & & & \\
\hline 117 & & & \\
\hline 117 & & & 10.3210 \\
\hline 100 & 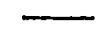 & - & 10.3314 \\
\hline 50 & - & 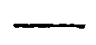 & $10 \cdot 3620$ \\
\hline 0 & 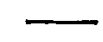 & $\longrightarrow$ & 10.3925 \\
\hline & zero & $\longrightarrow$ & $10 \cdot 4231$ \\
\hline 52 & & $\ldots$ & $10 \cdot 4243$ \\
\hline & & $\longrightarrow$ & $10 \cdot 4268$ \\
\hline
\end{tabular}

1. Then, if 10.4268 be the specific gravity of silver at $56^{\circ}$ below zero, and weighed in alcohol at that temperature, by calculation from its loss of weight at $\mathrm{C}$, it will appear to be $9 \cdot 796$, by taking 8141 as the supposed density of alcohol, dividing the quantity 1000 by the loss 83.1 , multiplying the quotient $12 \cdot 033$ by $\cdot 8141$, and dividing by $1 \cdot 000$, 
the specific gravity of water, the result of this being $9 \cdot 796$; What then is the specific gravity of the alcohol-if not 8141 ?

It is found by this rule :-

Divide 1000, the quantity weighed, by the loss of weight, and by the quotient divide the density of silver, according to its visible contraction; the quotient will be the specific gravity of the alcohol, or fluid in which it is weighed.

Thus, at the temperature $56^{\prime}$ below zero, the loss of weight is 83.1 ; by which, if $\cdot 1000$ (the quantity weighed) be divided, the quotient is $12 \cdot 033$, by which $10^{\circ} 4268$ (the density of silver according to its visible contraction) is divided ; and the quotient is -8665 , the specific gravity of the alcohol at $56^{\circ}$ below zero. Thus, 0524 is the sum of variation of the specific gravity of alcohol in 106, or 00049434 is the variation in each degree of temperature: from it the specific gravities at these several temperatures are calculated.

At $135^{\circ}$ the specific gravity of alcohol is $\cdot 77208$

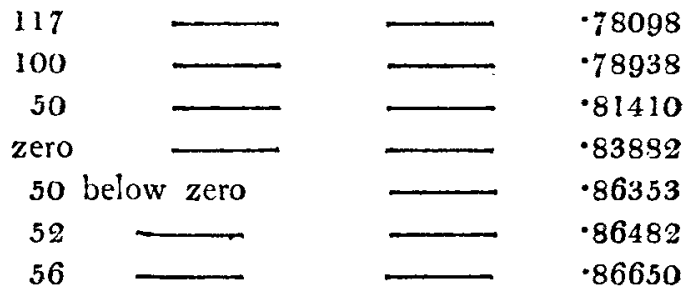

$\mathrm{K}$. Then taking the specific gravity of the alcohol at any certain temperature, and the loss expressed at $\mathrm{C}$; What will be the specific gravity of the silver according to $\mathrm{C}$ ?

It is found by this rule :-

Divide 1000 (the quantity weighed) by the loss of weight, and multiply the quotient by the specific gravity of the alcohol or fluid in which it is weighed.

Thus, at $56^{\circ}$ below zero the loss of weight is 83.1 , according to $\mathrm{C}$; by which if 1000 be divided, the quotient is 12.0337 ; which, multiplied by $\cdot 8665$, the density of alcohol, as at $\mathrm{I}$, in that temperature the product is 10.427 , the epecitic gravity of silver at $56^{\circ}$ below zero.

Then, having ascertained the specific gravity of silver and $\mathrm{Ka}$ 


\section{Un the Contraction which tokes place in Mercury}

of alcobol at several points of the scale of temperature by calculations at $\mathrm{I}$, from expcriments at $\mathrm{H}$, the calculation of the sperific gravity of mercury is thus performed :-

L.-Divide the quantity by the loss, and multiply the quotient by the specific gravity of the alcohol at such temperature.

Thus, mercury at $\mathrm{G}$, in the temperature of $56^{\circ}$ below zero, appears to be of the specific gravity $\mathbf{1 4} \cdot 465$. For dividing 1000 by 59.9 , the least loss in the solid state, and multiplying the quotient 16.694 by $\cdot 8665$, the specific gravity of alcohol at that temperature, the product is $14: 465$.

Then if mercury in $106^{\circ}$, from $50^{\circ}$ above to $65^{\circ}$ below zero, increases in specifie gravity from 13.613 to 14.465 , namely $\cdot 852$, which gives $\cdot 00804$ each degree; this sum, multiplied into the number of degrees from $50^{\circ}$ above zero, gives the specific gravity of mercury at that degree, if deducted when above 50 from 13.013 , or added to the same when below $50^{\circ}$.

Thus at $135^{\circ}$ above zero, which from $50^{\circ}$ is $83^{\circ}$, if the sum .00504 be multiplied by 85 , the product is $\cdot 6834$; which subtracted from 13.613 , the specific gravity of mercury at $50^{\circ}$, gives 12.9296 , the specific gravity of mercury at $135^{\circ}$ of temperature.

At $200^{2}$ the specific gravity of mercury is $12 \cdot 407$

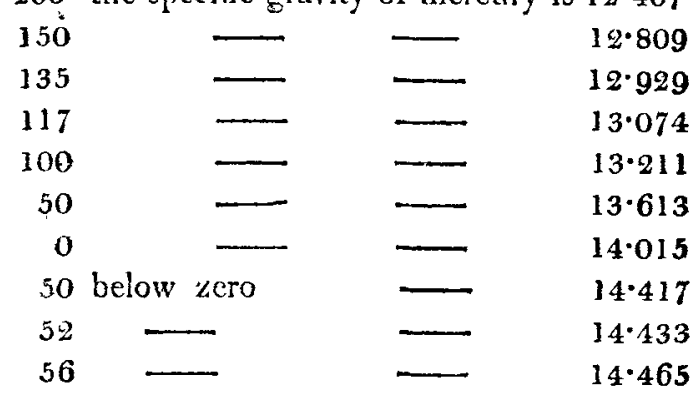

Then, to see if the alcohol in which the mercury was weighed at $G$ obtains by the same mode of calculation a specific gravity, according with the calculations at $I$, from the experiments at $\mathrm{H}$ on silver, - observe the rule at $\mathrm{I}$.

At the temperature $56^{\circ}$ below zero, the loss of weight is $59^{\circ} 9$ on the quantity 1000 ; by which if it be divided, 16.694 
is the quotient; this again dividing the specific gravity of the mercury 14.465 , the quotient is at this. temperature -8665 , the specific gravity of the alcohol according to $G$, which agrees with the calculations from the loss of weight of silver by experiments at $\mathrm{H}$, and calculations at $I$, it there appearing that $\cdot 8665$ is the specific gravity of the alcohol.

M. Supposing the mercury weighed by the hydrostatic balance at $\mathbf{G}$ in a solid form, during the variations in the loss of weight from 59.9 to 61 , to have passed through $20^{\circ}$ of temperature to $76^{\circ}$ below zero, and to have increased -001 each degree, then mercury in a solid state will have arrived at the specific gravity $14^{\circ} 485$, and the alcohol in which it is weighed with a loss of weight of 61 will consequently be of greater specific gravity.

For if alcohol at $56^{\circ}$ helow zero was of the specific grivity 8665 , as at $I$, the mercury losing $59^{\circ} 9$, as at $G$, and at a lower temperature the same mercury weighed in the same alcohol lost 61 , it will appear that the alcohol must have increased in specific gravity; for if 59.9 be $\cdot 8665,61$ will be .8842 , the mercury remaining at 14.465 , as at $\mathrm{L}$ : but as we have reason to state, it passes on to 14.485 at $76^{\circ}$ bclow zero, then the specific gravity of the alcohol must appear to be $\cdot 8854$; because if 14.485 increases $\cdot 001$ each degree, .8542 will increase 00006 ; ; which sum, multiplied by 20 , equals $\cdot 001220$; this, added to $\cdot 8842$, equals $\cdot 8854$.

$\mathrm{N}$. We observe at $F, 3024$ of mercury lose 222.1 at $50^{\circ}$ of temperature; at $200^{\circ}$ of temperature it loses $217 \cdot 4$; and by calculations at $L$, the specific gravity of the mercury so weighed was shown to be 12.407 at that degree of heat.

Then by the rule at $\mathrm{I}:-$

If 3024 be divided by $217 \cdot 4$, the quotient is $13 \cdot 000$, which sum dividing 12.407 , the known gravity of mercury at $\mathrm{L}$, the specific gravity of the water will consequently appear to be $\cdot 8920$ : this deducted from 1000 , the specific gravity of water at $50^{\circ}$ of temperature, leaves 1080 , the sum of differrence in 150 degrees, and 
150 On the Contraction which takes place in Mercury

At the following temperatures water is of the specific gravity stated: $200^{\circ}$

$\begin{array}{rlr}150 & & \\ 135 & -9306 \\ 117 & =9419 \\ 100 & = & 9537 \\ 50 & -9661 \\ 1 \cdot 0000\end{array}$

and as 1080 is the sum of difference in $150^{3}$, this sum $\cdot 000 \% 2$ expresses nearly the variation in each degree.

We find from the data obtained in these experiments a ratio of contraction between mercury, alcohol, water, and silver of equal volume.

$\begin{array}{ll}\text { Mercury at } \mathrm{L} \text { is } & \cdot 00804 \\ \text { Alcohol at } \mathrm{I} \text { is } & \cdot 0004943 \\ \text { Water from } \mathrm{F} \text { above } & \cdot 0007200 \\ \text { Silver at } \mathrm{H} \text { is } & \cdot 0006114\end{array}$

What will be the proportionate contraction of each, that of mercury being supposed 100 ?

As the increased specific gravity of mercury is to the supposed number, so is the increased specific gravity of either to the number required. If 00804 be 100 , what will $\left\{\begin{array}{l}0004943=6 \cdot 144 \text { Contraction of alcohol } \\ 0037200=8 \cdot 955 \quad \text { water } \\ 0006114=7 \cdot 604\end{array}\right.$ of equal volume, when that of mercury is 100 .

If we suppose water to be 1.0000

then mercury will be $11 \cdot 1666$

$\begin{array}{ll}\text { alcohol } & \cdot 6865 \\ \text { silver } & .8491\end{array}$

As in the bydrostatic balance the comparison relates to equal volumes of the thing weighed, and the fluid in which it is weighed; What will be the ratio of contraction of equal weights of each of the above?

As the specific gravity of either is to the specific gravity of mercury, so is the ratio of contraction of equal volumes to the ratio of contraction of equal weights.

As $-8141: 13613:: 0004943=008265$ alcohol.

As $1 \cdot 000: 13613:: 0007200=009801$ water.

As $10.362: 13613:: 0006114=000803$ silver.

008040 mercury.

Table 
at low Temperatures.

Table of Results of the foregoing Experiments, with some Calculations from them.

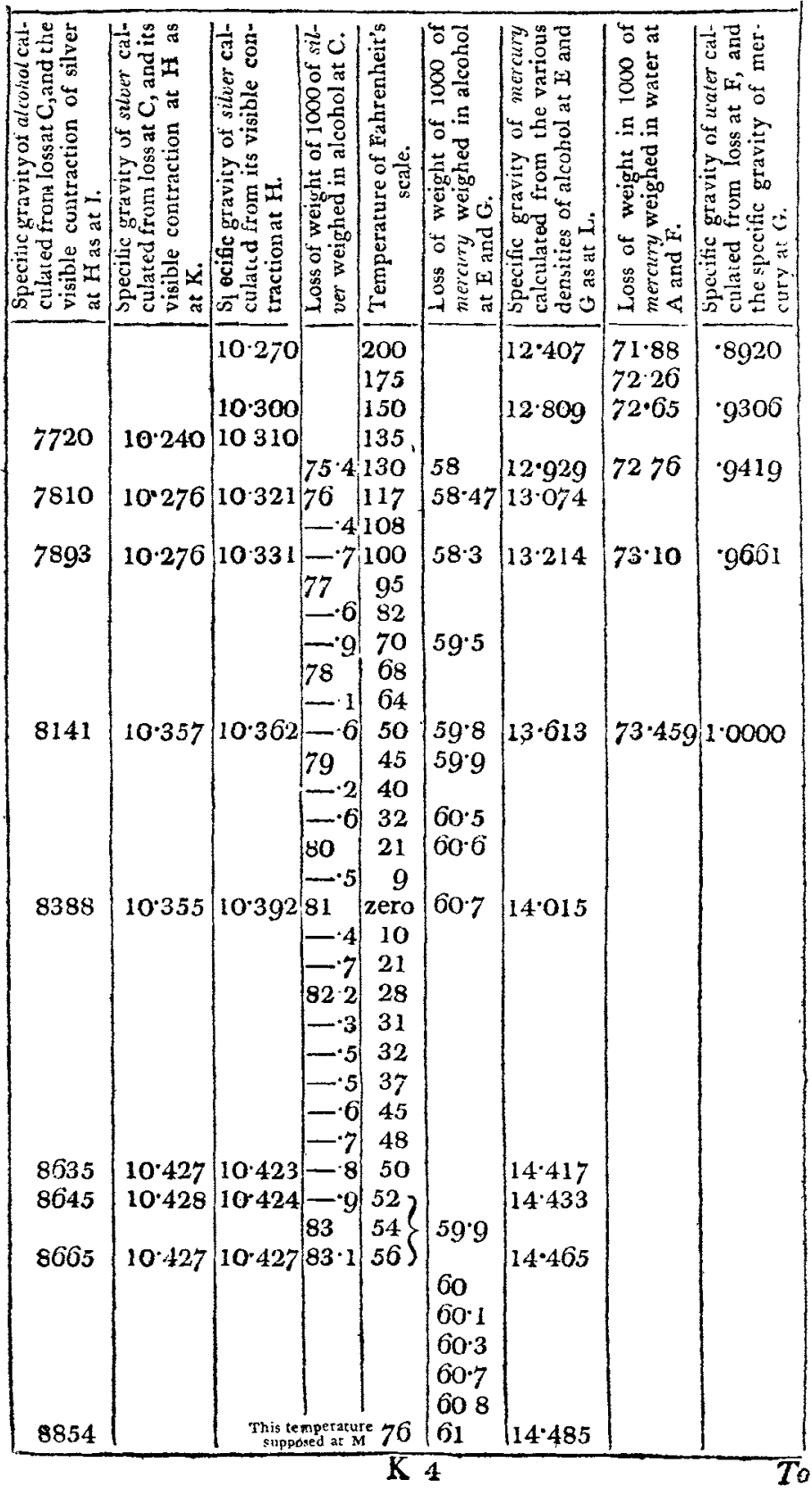


To recapitulate the foregoing Experiments-

Observe,

At A, mercury is of the specific gravity 13.613 at the temperature 50 .

At $B$, alcohol is of the specific gravity .8141 at the temperature 50 .

At $C$, silver is of the specific gravity 10.362 at the temperature 50 .

At $C$ and $D$ there is error shown to exist in estimating the specific gravity of bodies in the usual mode, without having regard to a fixed point of temperature, at which the medium chosen to compare other bodies with should be estimated at 1.000 ; and without having regard to the ratio of contraction in the body weighed, and the medium in which it is weighed.

At $E$, a variation of contraction between merpury and alcohol expressed by 22 in weighing 5276 of mercury.

At $F$, the error of common practice noted at $D$ is confirmed, the increased loss of weight shawing the contraction of water to be greater than that of mercury of equal volume; it is expressed by 4.7 in $150^{\circ}$ on the quantity 3024.

At $G$, mercury in its frozen state weighed by the hydrostatic balance lost 59.9 to 61 on the quantity of 100 . For the calculations from these facts, see $\mathrm{I}$, and the annexed table. The mercurial thermometer fell below $270^{\circ}$ on the scale below zero.

At $\mathrm{H}$, silver, going down 150 degrees of Fabrenheit's scale, contracts $\frac{1}{3 \frac{1}{4}}$ th part of its dimensions, which is called its visille contraction.

The silver at 117 above zero is 10.321

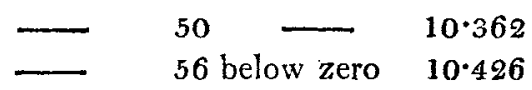

The increase of silver in its specific gravity is $\cdot 0006114$ each degree.

At $I$, the error of general practice observed at $D$ and $F$ is confirmed. A rule is given for finding the specific gravily of the alcohol. When 8141 at 50 above zero it appears to be .8665 at 56 below zero. 
At I, the increased specific gravity of alcohol each degree is 0004934 .

For the further variations of gravity see the annexed table. At $\mathrm{K}$, a rule for finding the specific gravity of silver from loss of weight at $\mathrm{C}$, and the specific gravity of alcohol at $\mathrm{I}$.

If silver at 50 above zero be $10 \cdot 362$, it will be at 56 below zero 10.427 .

At $\mathrm{L}$, a rule is shown for finding the specific gravity of mercury.-At 200 above zero it is found to be $\mathbf{1 2 \cdot 4 0 7}$

50

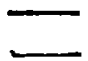

$13 \cdot 613$

$14 \cdot 465$

The increase each degree is $\cdot 00804$

A comparison of $\mathrm{C}$ and $\mathrm{G}$ as it relates to alcohol; and by the rule at $I$ it appears they accord in the number $\cdot 8665$, the specific gravity at 56 below zero.

At $M$, the contraction of mercury in its solid state, supposed to be near twice that of silver, or $\cdot 001$ each degree, because near the point of fluidity.

Alcohol of the specific gravity $\mathbf{8 8 5 4}$ at 76 below zero.

Mercury in a solid form of the specific gravity 14.485 at 76 below zero.

The mode of calculating this is shown.

The increase of specific gravify of alcohol in each degree not shown by the hydrostatic balance, when mercury is weighed in it in a solid form, is .000061 .

At $N$, rule for finding the increase of water of equal bulk to that of mercury. It appears from $F$ to be $\cdot 00072$ each degree.

Rule for finding the ratio of contraction of mercury, alcohol, water, and silver, of equal volume; mercury being supposed 100 .

A ratio of contraction also of water, mercury, alcohol, and silver; water being supposed $1 \cdot 000$.

Rule also given to find the ratio of contraction of alcohol, water, silver, and mercury, of equal weight. 